\title{
QUELQUES RÉFLEXIONS SUR LA POÉSIE FRANÇAISE DE LA SECONDE GUERRE MONDIALE
}

Pendant la Seconde Guerre mondiale en France certains poètes estiment qu'ils ont un rôle social considérable et se font les porte-parole des opprimés. Ils s'engagent dans la lutte corps à corps mais aussi la plume à la main, tout en élevant leur voix pour proclamer leur foi en la liberté et pour montrer que leurs vers patriotiques peuvent devenir une arme virulente contre l'occupation nazie et les souffrances du peuple. Les poètes deviennent donc messagers de paix et d'espoir et les combattants apprennent leurs poèmes par cœur ou on les diffuse légalement ou clandestinement. Mais il est possible de voir les choses sous un autre angle : il existait un certain nombre de poètes qui refusaient tout engagement littéraire, comme c'était le cas des anciens surréalistes, de même, il y avait des poètes qui s'engageaient du côté de la Collaboration, ou ceux qui refusaient de publier ou ne pouvaient même pas le faire sous l'Occupation pour des raisons diverses : matérielles, morales ou autres. Nous nous proposons d'aborder cette problématique, certainement très vaste et complexe, en prenant en considération les contraintes d'un article.

Mots clés : poésie française du XXe siècle, poésie engagée, poésie sous l'Occupation, poètes de la Seconde Guerre mondiale, poètes de la Résistance

\section{INTRODUCTION}

La poésie est souvent associée à une expression de soi et en général elle est intime, puisqu'elle sert à exprimer des sentiments personnels. Mais au cours des temps agités elle change sa tonalité et s'oriente vers l'Histoire. Par exemple, Victor Hugo, le plus grand poète du XIXe siècle, a considéré le poète comme un guide qui devait conduire le peuple vers le progrès, et c'était sa raison personnelle de prendre part, très activement, à la vie politique en France et d'écrire des vers qui serviront d'arme tranchante contre le régime actuel, celui de l'empereur Napoléon III. Ainsi, ses Châtiments, parus en 1854,

\footnotetext{
*diana.popovic@ff.uns.ac.rs
} 
sont-ils considérés comme un des recueils ayant réussi à orienter la poésie française vers une direction qu'on appelle, généralement, l'engagement. ${ }^{1}$

Quant au vingtième siècle, on peut remarquer que la poésie en France et également au monde entier - suit de près les turbulences de l'Histoire immédiate, surtout pendant les grands conflits mondiaux tels que la Grande Guerre, la Seconde Guerre mondiale, la guerre civile espagnole, la guerre civile grecque ou bien le combat pour la décolonisation, dont le but commun est toujours le même : la liberté du peuple. Alors les poètes révoltés prennent leur position qui n'est plus uniquement indivuduelle, mais plutôt sociale et c'est la raison pour laquelle ils se cachent derrière un nous. Citons ici le fameux fragment 131 de Feuillets d'Hypnos de René Char : "À tous les repas pris en commun, nous invitons la liberté à s'asseoir. La place demeure vide mais le couvert reste mis » (Char, $1983: 206)$.

Et comme Eugène Guillevic dit dans son poème "Art poétique », publié en 1942 dans le recueil Terraqué, c'est aux poètes de s'exprimer, même si c'est « un cri difficile / à former dans la gorge» (Guillevic, 2001 : 139). Les mots sont vivants, et si le bourreau, c'est-à-dire l'occupant nazi, veut étouffer les poètes, il ne réussira jamais, puisque les poètes savent comment modeler les mots: "Mieux que la main du menuisier / Avec le bois.» (vers 33-34) (Guillevic, $2001: 140$ ).

\section{LE CONTEXTE HISTORIQUE}

L'occupation du pays à partir de juillet 1940, divise le territoire en deux zones. L'instauration du régime de Vichy et son entrée dans la voie de la collaboration divisent les Français : la plupart voient en Vichy le salut, d'autres non, en fait, ils soupçonnent le pire. Puis, la répression adoptée par le régime prépare une collaboration ouverte et l'occupation totale du pays, à partir de novembre 1942, ce qui nourrit ces soupçons à l'égard de Vichy et se transforme en une défiance et fait naître la Résistance française. Au cours de la période noire de l'Occupation, la littérature entre en clandestinité.

${ }^{1}$ N'oublions pas ici toute une littérature révolutionnaire qui précède l'engagement hugolien, parmi lesquels se trouvent les œuvres d'un Jean-Baptiste Clément (18361903), participant au mouvement populaire de la Commune, qui est brutalement réprimé par le gouvernement conservateur d'Adolphe Thiers. Son poème La semaine sanglante, qui rappelle cet épisode épouvantable, date de 1871. 
Certains poètes estiment qu'ils ont un rôle social considérable et se font les porte-parole des opprimés. Ils risquent leur vie puisqu'ils s'engagent dans la lutte corps à corps mais aussi la plume à la main, tout en élevant leur voix pour proclamer leur foi en la liberté et pour montrer que leurs vers patriotiques peuvent devenir une arme virulente contre l'occupation nazie et les souffrances du peuple. Les poètes deviennent donc messagers de paix et d'espoir et les combattants apprennent leurs poèmes par cœur ou on les diffuse légalement ou clandestinement. Il s'agit donc d'une poésie qu'on considère patriotique, engagée, poésie de la Résistance. Mais, qu'est-ce que la Résistance ? D’après René Char : «Résistance n’est qu'espérance. Telle la lune d'Hypnos, pleine cette nuit de tous ses quartiers, demain vision sur le passage des poèmes » (Feuillets d'Hypnos, fragment 168).

La Résistance désigne l'action de s'opposer à l'occupation allemande. Le général de Gaulle utilise ce mot dans son appel prononcé le 18 juin 1940 sur les ondes de la radio anglaise, la BBC, et il exhorte tous ceux qui pouvaient le rejoindre en Angleterre, en disant: "Quoi qu'il arrive, la flamme de la résistance ne doit pas s'éteindre et ne s'éteindra pas » (Bervas-Leroux, 2006 : 5). Ce mot est largement accepté en France par les groupes qui s'opposent à l'occupation du pays par les troupes allemandes. Le Musée de l'Homme a publié clandestinement une publication dont le titre était justement Résistance. ${ }^{2}$ Dès 1942, le mot s'écrit avec une majuscule, tout en désignant l'ensemble de ceux qui s'engagent dans la Résistance (des résistants), des organisations et des mouvements qui y participent.

La Résistance est basée sur un refus très fort: d'abord sur le refus d'accepter l'armistice, le refus d'accepter l'occupation du pays par les Allemands et également le refus de collaborer avec l'occupant et le régime de Vichy. Une partie de la lutte contre l'ennemi était une lutte des mots, puisqu'il fallait réveiller le patriotisme et redonner de l'espoir. Des feuillets imprimés que l'on a distribués ou collés et qu'on appelait les papillons, ou d'autres brochures de propagande, feuilles ou tracts, ont été imprimés en cachette. Posséder ces papiers interdits signifiait le danger d'être arrêté. Des écrits ou des poèmes sont donc appris par cœur ou recopiés et diffusés «sous le

${ }^{2}$ C'était en souvenir d'une protestante qui était prisonnière au cours des guerres de religion, qui a gravé ces lettres sur les murs de sa cellule. 
manteau ». Les papiers qui étaient destinés à être publiés légalement, étaient pourvus de double sens - c'était une "poésie de contrebande ».

La parole poétique était destinée au peuple. C'était toute une armée de gens rebelles qui ont volontiers élevé leur voix pour protéger la valeur suprême qu'était la liberté. Cette poésie de lutte et d'espoir voulait rendre les "hommes frères des hommes", contre la "bêtise et la démence », comme l'écrit Paul Éluard dans son poème "Un feu sans tache », de 1941. Cette poésie et leurs auteurs étaient sous une menace permanente: une menace d'emprisonnement ou même de mort. ${ }^{3}$ Donc, écrire signifie risquer sa liberté ou risquer même sa vie, ce qui implique d'être rusé et de faire des détours pour échapper à la censure et à des représailles. C'est la raison pour laquelle René Char a pris la décision de ne plus écrire de poèmes entre 1941 et 1944, lui qui tenait un rôle décisif dans la Résistance active. Au cours de cette période le capitaine Alexandre (son pseudonyme de guerre) consigne des notes dans un carnet où il témoigne de son engagement. Ces textes, sous forme d'un journal sont intitulés Feuillets d'Hypnos et ne seront publiés qu'après la guerre : "Ces notes marquent la résistance d'un humanisme conscient de ses devoirs, discret sur ses vertus, désirant réserver l'inaccessible champ libre à la fantaisie de ses soleils, et décidé à payer le prix pour cela. » (Char, 1983 : 173, souligné par D. P.).

Dès 1940, donc avant que la Résistance ne s'organise, les poètes se regroupent et leurs œuvres deviennent de véritables armes. Ils se réunissent, en zone libre, autour de la revue Poésie, publiée par Pierre Seghers, une revue à laquelle ont contribué Aragon, Éluard et Jouve. On se regroupe aussi autour la revue lyonnaise de René Tavernier, Confluences. À Alger, Max-Paul Fouchet fonde Fontaine. C'est un espace de liberté - «champ libre» mentionné par Char - pour les écrivains et intellectuels, au temps où la presse demeure aux mains du pouvoir et ne s'occupe que de sa propagande. La poésie, donc, doit se découvrir une nouvelle fonction, celle de mettre en circulation des vers, des formules, des refrains, des maximes, des images et tout ce qui peut sauver

${ }^{3}$ Par exemple, Jean Cassou a été incarcéré pendant dix-huit mois pour la propagande anti-vichyste ; le cofondateur des Lettres françaises a été arrêté et fusillé au lendemain de la parution du premier numéro de la revue ; en 1942 la Gestapo a brûlé le manuscrit de La Lutte avec l'ange d'André Malraux qui rejoint la Résistance en 1943 et à partir de 1944 il commande, sous le nom de colonel Berger, les Forces françaises libres du Lot. 
l'honneur du peuple. Tous ces écrits sont publiés sous forme de tracts ou de feuillets insérés dans des revues. Par exemple, le poème le plus célèbre d'Éluard, "Liberté », qui date de 1942, a été parachuté en milliers d'exemplaires par l'aviation anglaise, la RAF (Royal Air Force), sur les territoires occupés, pour encourager les résistants. Le poète lui-même a confirmé que le but en était de « retrouver, pour nuire à l'occupant, la liberté d'expression » (Éluard, $1968:$ 1607-1608) :

XIX Sur l'absence sans désir

Sur la solitude nue

Sur les marches de la mort

J'écris ton nom...

XXI Et par le pouvoir d'un mot

Je recommence ma vie

Je suis né pour te connaître

Pour te nommer

Liberté. $^{4}$

C'étaient des gens de l'ombre dont les paroles exprimaient la colère contre toute sorte de souffrance de la nation, contre la répression, l'occupation nazie et l'asservissement du peuple, ou c'était en revanche une expression du désarroi de la débâcle. C'étaient des paroles de contestataires, critiques, très souvent écrits sur un ton satirique. Quelquefois c'était une poésie anonyme ou celle qui réutilise les vers des poèmes connus pour les modifier et placer dans un autre contexte. En tout cas, il s'agissait d'une «action verbale» qui se voulait utile, encourageante pour les guerriers. Et les poètes étaient fiers d'avoir pu y participer. Cela est visible même dans le titre d'un recueil collectif de poèmes engagés, L'Honneur des poètes, publié en 1943 par les Éditions de Minuit clandestines dans le cadre de la Résistance (Garnaud, 2013 : 109),

${ }^{4}$ Ce poème a été traduit en plus de dix langues, et aussi mis en musique par Francis Poulenc (Lacombe, 2013). L'histoire de ce poème est intéressante, puisqu'il était d'abord destiné à Nush, femme aimée du poète, et était d'abord intitulé "Une seule pensée ». Mais puis, le poète a décidé d'ajouter à la fin un vers supplémentaire consistant en un seul mot, «Liberté », ce qui a changé complètement la signification du poème : d'un chant d'amour, il est devenu un chant de révolte. (Éluard, 1968 : 1608). 
auquel ont contribué, entre autres, Louis Aragon et Paul Éluard. ${ }^{5}$ Mais, vu le fait qu'ils étaient surréalistes, prêter sa plume à une cause juste, à un peuple, est-ce devenir traître à sa vocation et à son idéal de l'art ? Aux yeux de Benjamin Péret, un des fondateurs du surréalisme, la réponse est affirmative. Malgré son hostilité aux fascismes, il est toute sa vie resté fidèle aux conceptions esthétiques du mouvement surréaliste. Vers la fin de 1941 il s'exile au Mexique et c'est à Mexico qu'il publie, en 1945, un pamphlet, Le Déshonneur des poètes, où il estime que certains poètes se sont fourvoyés et il déplore qu'ils se soient laissés au piège du patriotisme, du nationalisme ce qui signifiait une certaine réhabilitation de la religion. Pour lui, il s'agissait d'une trahison envers l'idéal poétique. Leurs vers étaient d'après lui d'un «niveau lyrique de la publicité pharmaceutique » (Péret, 1945, para. 12). Lui, il est contre tout contrôle de la raison, contre ce "classicisme» de la langue, contre toutes contraintes traditionnelles et formelles, tellement éloignées de l'écriture automatique si pratiquée autrefois. Donc, il ne reconnaît plus ses amis d'avant-guerre (qui sont devenus "camarades ») qui maintenant exploitent les misères du peuple pour nourrir leur imagination et leurs œuvres didactiques, qui, ne pourraient jamais être considérées, d'après lui, comme une bonne littérature.

Pas un de ces «poèmes» ne dépasse le niveau lyrique de la publicité pharmaceutique et ce n'est pas un hasard si leurs auteurs ont cru devoir, en leur immense majorité, revenir à la rime et à l'alexandrin classique. La forme et le contenu gardent nécessairement entre eux un rapport des plus étroits et, dans ces "vers ", réagissent l'un sur l'autre dans une course éperdue à la pire réaction. Il est en effet significatif que la plupart de ces textes associent étroitement le christianisme et le nationalisme comme s'ils voulaient démontrer que dogme religieux et dogme nationaliste ont une commune origine et une fonction sociale identique. Le titre même de la brochure, L'Honneur des poètes, considéré en regard de son contenu, prend un sens étranger à toute poésie. En définitive, l'honneur de ces 'poètes' consiste à cesser d'être des poètes pour devenir des agents de publicité. (Péret, 1945, para. 12).

De ce même côté on retrouve écrivain et journaliste anglais Arthur Koestler, qui dit, lui aussi, que la poésie de la Résistance, surtout d'Aragon et de

${ }^{5} \mathrm{Il}$ y avait plusieurs ouvrages avec le même titre aussi bien que plusieurs éditions où les poètes se cachaient derrière les pseudonymes inventés par Éluard. 
Vercors, «n'est que charlatanisme littéraire » (Bervas-Leroux, 2006 : 8). Donc, certains auteurs sont contre tout engagement littéraire, vu comme utilitaire, conçu comme utilitaire, voire nuisible pour une écriture libre dont l'imagination doit rester libérée de la logique et de la raison.

\section{LES POÈTES « ÉGARÉS »}

Cette trahison poétique dont parle Benjamin Péret, n'a rien avoir avec une trahison idéologique. Sous l'Occupation il y avait des écrivains qui ont résolument épousé l'ideologie nazie et qui demeuraient du côté du régime de Vichy. C'est le cas de Robert Brasillach, l'auteur du roman La Conquérante, paru en 1943. Il était rédacteur en chef du journal collaborationniste et antisémite intitulé Je suis partout. Il est ensuite jugé pour ses écrits politiques, condamné et fusillé durant l'épuration, le 6 février 1945, à l'âge de 35 ans. Mentionnons aussi Pierre Drieu La Rochelle, l'auteur de Gilles (1939), qui était également fasciné par le totalitarisme allemand. Il était un auteur plus complexe et plus ambigu que Brasillach. Il a dirigé la Nouvelle revue française de 1940 à 1943. Il se suicidera en 1945. Puis, son ami Abel Bonnard, poète et écrivain, mais aussi homme politique - il est nommé ministre de l'Éducation nationale en 1942 qui fait partie des « ultras » et des derniers partisans du régime de Vichy qui se réfugient à Sigmaringen en 1944. Son collaborationnisme et le soutien qui lui est apporté par Otto Abetz, ambassadeur allemand en France et son ami, lui valent le surnom d'« Abetz Bonnard ». Ils sont tous culturellement germanophiles. Les huit écrivains français, parmi lesquels Pierre Drieu la Rochelle, Marcel Jouhandeau, Robert Brasillach, Jacques Chardonnes, répondent à l'invitation de Joseph Goebbels, ministre de la Propagande du Reich, en octobre 1941, et séjournent en Allemagne pour le Congrès des écrivains européens de Weimar, dont ils reviennent enthousiasmés, voire favorables à Hitler.

À part la trahison idéologique de certains poètes, il y avait une trahison de la part de jeunes femmes, devenues maîtresses des Allemands. Ces femmes, soupçonnées d'avoir été proches des nazis, on les tondait, ce que Paul Éluard décrit dans un passage de son carnet: «Réaction de colère. Je revois une magnifique chevelure féminine gisant sur le pavé. Je revois des idiotes lamentables tremblant de peur sous les rires de la foule » (Éluard, 1968 : 677). Son poème "Comprenne qui voudra", publié en 1944 dans le recueil $A u$ 
rendez-vous allemand, est consacré à une jeune femme qui est «Souillée et qui n'a pas compris / Qu'elle est souillée » (Ibid).

\section{LES GRANDS THÈMES}

En général, tous les grands thèmes de la poésie de la Résistance proviennent d'un sentiment de patriotisme très fort, qui se dévoile sous plusieurs aspects :

\section{Dénoncer la violence de l'oppresseur}

Il y a des poèmes où l'on parle de la souffrance et de la mort des combattants. Tel est « La Nuit de Mai ${ }^{6}$ de Louis Aragon, écrit en 1942, où le poète évoque l'échec de l'armée française en mai 1940. Ses quatrains donnent un portrait des soldats d'hier et d'aujourd'hui, tout en montrant que deux conflits mondiaux - la Grande Guerre et la guerre actuelle - se font écho dans la mémoire collective. Même si l'on traite d'un événement concret, on a l'impression qu'on parle d'une manière beaucoup plus générale.

Il y a des poèmes où l'on voit une explosion de la violence qui déborde toute imagination possible, puisqu'il ne s'agit plus des horreurs des tranchées, mais d'une violence planifiée, maîtrisée, concertée. C'est une sorte de désordre de la guerre : les arrestations au petit jour, les liquidations, la torture, les prises d'otage - tout cela se trouve dans des vers. Robert Desnos ${ }^{7}$ dans ses « Couplets de la rue Saint-Martin » parle de la perte de son ami André Platard - arrêté par les Allemands ou par les milices françaises qui collaboraient avec l'occupant qui devient symbole de tous les disparus au cours de l'Occupation allemande (Le Guern-Camara, $2011: 23$ ).

Mentionnons aussi que René Char décrit, d'une manière détaillée et suggestive, une scène de torture qu'on pouvait voir tous les jours dans les lieux où l'on supposait tomber sur des résistants. Il s'agit du fragment 128 des

\footnotetext{
${ }^{6}$ Le titre est allusion à un poème de Musset qui a le même titre et où l'on parle d'un mal-être intérieur et individuel, mais dans le poème d'Aragon il s'agit d'un autre temps et des problèmes collectifs.

${ }^{7}$ Desnos fournissait des faux papiers à des juifs et à des résistants. Il est arrêté par la Gestapo en 1944 et déporté au camp de Terezin en Tchécoslovaquie où il meurt le 8 juin 1945.
} 
Feuillets d'Hypnos où l'on peut voir les Allemands forçant un jeune homme à révéler le lieu de cachette du capitaine Alexandre.

\section{La colère du poète}

La plume devient égale à une épée pour combattre les injustices et laisse voir toute sa puissance qui se déploie par le Verbe. C'est la guerre des écrivains engagés où il faut exprimer non seulement leurs attitudes et convictions, mais aussi décrire des situations de terreur, situations extrêmes, toute une tragédie qui se mêle, naturellement, avec des sentiments exacerbés et véhéments. Le poète exprime sa rage, sa haine, puisque la souffrance et les spectacles de la mort dépassent la limitede l'imaginable. Les vers et d'autres textes sont pleins des sentiments très forts, susceptibles parfois de se muer en de vraies rafales de mitraillette.

Ainsi Robert Desnos décrit sa haine croissante dans son poème « $\mathrm{Ce}$ cœur qui haïssait la guerre... » en décrivant le battement non seulement de son cœur à lui, mais « de millions d'autres cœurs battant comme le [s]ien à travers la France », la haïne qui culmine dans ce cri : "Révolte contre Hitler et mort à ses partisans!» (Le Guern-Camara, 2011 : 24-25). Le poème, publié dans L'Honneur des poètes sous le pseudonyme de Pierre Andier, date de 1943, une année noire. Sous un autre pseudonyme, Valentin Guillois, Desnos écrit un poème sur ses activités clandestines, "Le veilleur du Pont-au-Charge », où il décrit, sans enjolivement, la manière dont il a neutralisé un ennemi. Il y évoque la vie quotidienne des résistants, il énumère leurs activités clandestines, associées à la violence et à la mort.

La haine envers la tyrannie de l'ennemi est très présente dans les vers de Paul Éluard. Parfois il chante sur un ton perçant et sarcastique, comme dans son poème "Bêtes et méchants", publié en 1945 dans Au rendez-vous allemand, où il donne un portrait caricatural des soldats allemands. En dehors de la raillerie délibérée des Allemands, il veut également livrer un message d'espoir pour encourager ses camarades de la Résistance.

Donc l'attaque du côté du poète, sans qu'il cherche à adoucir son discours, est un des aspects de la littérature de la Résistance. Elle réagit à la réalité horrible, pénible dans tous les sens, ce qui se reflète sur des expressions langagières, sur le ton que les poètes adoptent pour transmettre leurs témoignages et/ou transmettre leurs messages. 


\section{L'amitié et la solidarité}

Ce sont les sujets récurrents de la poésie de la Résistance, ce qui est compréhensible puisqu'on ne se trouve que dans un groupe de gens, autrement dit dans une communauté de combattants. En tant que valeurs, l'amitié et la solidarité sont associées aux actions non-individuelles qui procurent au groupe une force et une efficacité nécessaires pour la lutte.

Dans son poème "Avis $»^{8}$ Éluard, sous le pseudonyme de Jean du Haut, exprime la conviction d'un combattant que son combat singulier - même s'il terminera par sa mort qui se rapproche inéluctablement ${ }^{9}-$ ne sera pas inutile, et qu'il sera poursuivi de la vengeance et de la lutte des autres camarades. Le message est très puissant, puisqu'il parle de cette chaîne d'actions, cette multitude d'efforts qui vont assurément être couronnés par la victoire. Et cela sera la victoire du peuple entier, donc elle sera collective et non une victoire individuelle. Cette pensée apaisait l'esprit du jeune résistant et lui donnait de la force à la veille de sa mort lorsqu'« il a commencé à sourire » (vers 8). Donc sa mission se poursuit après lui.

Dans le poème mentionné, «Le veilleur du Pont-au-Change », Robert Desnos fait éloge de la fraternité entre les Alliés de tout le monde, qui mènent une même lutte contre l'oppression des nazis. Leur lutte globale pour la cause juste encourage «camarades de toutes nations » (vers 48), le jour et surtout la nuit, qui figure d'ailleurs comme le cadre temporel de ce poème.

D'un côté naissent une véritable haine et une violence envers l'ennemi, mais de l'autre côté naît un sentiment collectif, un amour de l'humanité, une fraternité parmi les camarades ce qui est vu comme un garant pour la future victoire commune. René Char exprime ce sentiment en disant: "J'ai aimé farouchement mes semblables cette journée-là, bien au-delà du sacrifice » (fragment 128 des Feuillets d'Hypnos, souligné par D.P.).

On était fier d'être ami, d'être camarade. On a fait des vers, mais parfois on les a mis en musique, pour les chanter. Cela était le cas d'un poème créé en 1943 sous le titre « Le chant des partisans », qui est devenu un véritable hymne de la Résistance en France, rédigé par deux écrivains français qui ont rejoint

8 Avis représente une liste d'otages que les nazis ont placardée sur les murs, pour effrayer le peuple et pour les dissuader d'adhérer à la Résistance.

${ }^{9}$ C'était le cas du jeune lycéen Lucien Legros exécuté en 1943 par les nazis. 
les Forces françaises libres du général de Gaulle en Angleterre, Joseph Kessel et Maurice Druon. En effet leur chanson a été inspirée d'un chant révolutionnaire russe. Leur version française a connu un grand succès mondial et une grande postérité. Elle est chanté par beaucoup d'interprètes tels qu'Yves Montand, Mireille Mathieu, Johnny Halliday et même par le groupe Zebda, qui l'a adaptée et en a changé le titre en "Motivés » (Le Guern-Camara, 2011 : 121). Ainsi, les mots, les messages, les sentiments étant fusionnés avec une musique vibrante et ils obtenaient ainsi une dimension supplémentaire pour toucher les cœurs.

Et lorsque la guerre s'achève au mois d'août 1945, on est heureux d'être vivant, mais le feu de la haine et de la douleur ne s'éteint pas. Ainsi voiton Éluard, qui a chanté autrefois son « Paris malheureux » sous l'occupation (vers 7 du " Courage ", écrit en 1943 sous le pseudonyme Maurice Hervent et publié en 1945 dans Au rendez-vous allemand), chanter dans «En plein mois d'août ", ce lundi soir victorieux où ceux qui se sont trouvés aux barricades finalement osaient montrer les yeux, et il n'oublie pas de remercier tous les résistants, en soulignant: :Que nos frères sont morts pour que nous vivions libres / Car vivre et faire vivre est au fond de nous tous / Voici la nuit voici le mirroir de nos rêves» (Éluard, $1968: 402$ ).

Le poème «La rose et le réséda » de Louis Aragon, publié en 1944 dans La Diane française et dédié à ses compatriotes qui ont été fusillés au cours de la guerre, exprime la nécessité que tous doivent être unis dans la même lutte pour la libération. Ce poème est un appel à l'union entre les communistes et les catholiques. L'image des fleurs, dont les couleurs, le rouge de la rose et le blanc du réséda, symbolisent des couleurs des communistes et des monarchistes catholiques, lui a permis de déjouer la censure.

Certes, il existe beaucoup de poèmes qui rendent hommage à ceux qui ont donné leur vie pour le peuple entier. Aragon, sous le pseudonyme de Jacques Destaing, dans sa "Ballade de celui qui chanta dans les supplices", écrite en 1943 et publiée dans La Diane française en 1944, décrit son camarade Gabriel Péri qui est mort en chantant "La Marseillaise», hymne national français. Et tout le poème a un rythme pour être chanté sur l'air de cette chanson fortement symbolique. 


\section{La vie, l'amour}

Pendant l'occupation, le désir d'avoir une vie libre et heureuse dans un pays libre est tout à fait légitime et avant tout humain. Il y a des vers qui chantent l'amour. En le chantant et louant en temps de guerre c'est aussi une manière de se révolter contre l'asservissement et de proclamer la liberté de vivre et d'aimer. Ainsi fait Éluard, par exemple. Il évoque une situation quotidienne pendant la guerre et c'est le couvre-feu, qui force les gens d'être enfermés. Lorsqu'il est interdit de sortir, le poète défie l'occupant dans une série de questions qui commencent par: "Que voulez-vous la porte était gardée / Que voulez-vous nous étions enfermés » et finit par «Que voulez-vous nous nous sommes aimés » (publié d'abord dans Poésie et vérité, en 1942, repris dans Au rendez-vous allemand, 1945).

De l'autre côté, Louis Aragon appartient aux poètes qui étaient fortement conscients que leurs vers provoqueront une réaction chez les ennemis (les nazis ou les collaborrateurs). Il évoque ses sentiments dans un de ses poèmes intitulé " Plus belle que les larmes », qui date de 1942, et est entré au recueil Les Yeux d'Elsa, en parlant d'une manière provocante (Le GuernCamara, $2011: 34)$ :

Quand je parle d'amour mon amour vous irrite Si j'écris qu'il fait beau vous me criez qu'il pleut Vous dites que mes prés ont trop de marguerites Trop d'étoiles ma nuit trop de bleu mon ciel bleu ...

Il est évident que le poète s'identifie avec sa patrie occupée et éprouve de très fortes émotions envers elle. Il s'agit d'un amour que l'occupant ne pourra jamais étouffer.

\section{LA POÉSIE EN TEMPS APOCALYPTIQUES}

Tout le monde lit la poésie, même les déportés. C'est leur nourriture spirituelle qui devient une nécessité, comme le pain quotidien. Dans un camp de concentration allemand un poète et lieutenant, Jean Bénac, écrit une lettre à Pierre Seghers, en 1942, en disant : «Votre revue me fait du bien. C'est tout. Il faudrait que tous les Français connaissent la place que nous donnons à la Poésie, nous qui n'avons rien, et la foi que nous avons dans la Poésie française » (Seghers, 2004 : 138). C'est un témoignage qui s'ajoute à d'autres 
écrits, tel que L'écriture ou la vie, un ouvrage autobiographique de Jorge Semprun où il décrit comment la poésie lui était importante pour survivre, à lui particulièrement mais comme à ses camarades, dans l'horreur de Buchenwald, camp de concentration près de Weimar, en Allemagne.

Cela nous donne la certitude que cette union entre ceux qui écrivent et ceux qui lisent était essentielle au temps de la détresse. Elle créait une communauté d'esprits et d'énergies nécessaire pour remporter la victoire et continuer à vivre. Le poète sonne la diane du poiein pour éveiller les soldats, exhorter à la révolte, ou pour rendre hommage à ceux qui sont tombés et les sauver ainsi de l'oubli.

\section{EN GUISE DE CONCLUSION}

La poésie a toujours été à l'écoute de son temps, de ses remous historiques. Contre l'envahisseur, on s'est battu avec des mots. La poésie était une arme très forte et privilégiée au service de l'intégrité nationale. Les poètes témoignaient ou résistaient. Pourtant, ils ne pouvaient pas diffuser leurs œuvres sans difficultés, puisque le papier était une denrée rare et la censure était constante. Les poèmes qui exprimaient un désir de liberté, de résistance, de colère, de combat et ainsi de suite, étaient appris par cœur ou recopiés et distribués sur des tracts.

Les recueils poétiques s'éclipsaient au bénéfice des revues telles que Poésie, Fontaine ou Confluences, dont l'impact était considérable. Éluard, Aragon et Char étaient incontestablement les figures de proue de la poésie de la Résistance (Maulpoix, 2009). Leurs œuvres encourageaient les gens et diffusaient un message d'espoir, de liberté, de vision d'un avenir plus prometteur. René Char l'affirme en écrivant ses mots qui se rapprochent d'un aphorisme: "À chaque effondrement des preuves le poète répond par une salve d'avenir » (Fureur et Mystère, 1948). Et lorsque l'avenir vient, on se souvient. "Strophes pour se souvenir » de Louis Aragon semble être un titre emblématique ${ }^{10}$. Vu son contenu et également son titre, ce poème, comme bien d'autres, rend un message humain puissant.

10 Ce poème date de 1955 et sera publié un an plus tard dans Le Roman inachevé. Aragon y ressuscite le sinistre souvenir de «l'Affiche rouge» de 1944, lorsque les 
Diana Popović

SOME REFLECTIONS ON FRENCH POETRY DURING WORLD WAR II

Summary

During World War II in France some poets considered themselves to have a significant social role and became porte-paroles of the oppressed. They took part in hand-to-hand fight, but also with a pencil in hand, raising their voices to declare their faith in freedom and show that their patriotic verses could become a deadly weapon against the Nazi occupation and the suffering of the people. The poets therefore became messengers of peace and hope, and the fighters memorized their poems or distributed them legally or secretly. But it's possible to look at things from a different angle: there were a number of poets who refused any literary engagement, as was the case with the former Surrealists, then there were poets engaged on the side of the Collaboration, and there were those who refused to or even they couldn't publish their works under the Occupation for various reasons: material, moral or other. This paper deals with this topic, which is in fact very large and complex, taking into account the limitations of the article.

Key words: $20^{\text {th }}$ century French poetry, committed poetry, poetry under the Occupation, World War II French poets, poets of Resistance.

\section{RÉFÉRENCES BIBLIOGRAPHIQUES}

Bervas-Leroux, A. (2006). Au nom de la liberté. Poèmes de la Résistance. Paris : Flammarion.

Char, R. (1983). OEuvres complètes. Introduction de Jean Roudaut. Paris : NRF Gallimard.

Ducey, B. (2008). La Résistance en poésie. Des poèmes pour résister. Paris : Magnard.

Éluard, P. (1968). CEuvres complètes. Tome 1. Édition établie par Marcelle Dumas et Lucien Scheler. Paris : Gallimard.

Enseigner la Résistance (2020). Consulté le 12 octobre 2020, disponible surhttps://www.reseau-canope.fr

vingt-trois partisans ont été fusillés. Léo Ferré va mettre ces vers en musique en 1959, sous le titre de L'Affiche rouge. 
Garnaud, D. (2013). Les mutations de la rhétorique dans l'écriture de Guillevic. (Thèse de doctorat). Bordeaux : Université Michel de MontaigneBordeaux 3.

Guillevic, E. (2001). Art poétique. Précédé de Paroi et suivi de Le Chant. Paris : Gallimard.

Lacombe, H. (2013). Francis Poulenc. Paris : Fayard.

Le Guern-Camara, G. (2011). La Poésie engagée. Paris : Belin - Gallimard.

Maulpoix, J.-M. (2009). Pour un lyrisme critique. Paris : José Corti.

Péret, B. (1945). Le Déshonneur des poètes. Paris : José Corti. Consulté le 12 octobre 2020, disponible sur https://www.benjaminperet.org/benjamin-peret/extraits-de-loeuvre/13-la-revolution/61-ledeshonneur-des-poetes.html

Poésie de la Résistance. In: Coupedouble.com. Consulté le 12 octobre 2020, disponible sur http://www.copiedouble.com/content/poésie-de-larésistance

Sghers, P. (2004). La Résistance et ses poètes. Tome 1. (France 1940/1945). Paris : Éditions Seghers. 
